\title{
Verwaltungsethnographie aus der Perspektive aktivistischer Forschung
}

\author{
Rezension zu Lisa Riedner (2018): Arbeit! Wohnen! Urbane Auseinandersetzungen um \\ EU-Migration. Eine Untersuchung zwischen Wissenschaft und Aktivismus. Münster: \\ edition assemblage.
}

Jenny Künkel

Abb. 1 Titel des Buches (Quelle: Verlag edition assemblage)

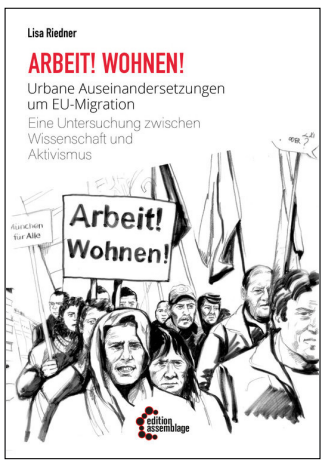

Aufregung herrscht unter einer Gruppe aus Tagelöhner*innen, vorrangig aus EU-Ländern, und den ehrenamtlichen Mitarbeiter*innen eines Workers' Center, als die Polizei aufkreuzt. Hektisch verhindert eine junge Wissenschaftlerin, die beim Center aktiv ist, dass die Beamt*innen auf einen undokumentierten Kollegen aufmerksam werden. Dieser jedoch steigt zu ihrer Verblüffung in den Polizeiwagen - hat er doch längst begriffen, wie er den Polizeikontakt als ersten Schritt zum Asylantrag (statt zur Abschiebung) nutzen kann.

Mit der nötigen Selbstreflektion und einer gehörigen Portion Selbstironie beschreibt Lisa Riedner die kollektiven Lernprozesse und gemeinsamen politischen Aktionen aus ihrer Zeit als Aktivistin bei einem Münchner Workers' Center, die sie zum empirischen Gegenstand einer Promotion machte. In ihrer Dissertation untersucht die Kulturanthropologin die sozialen Kämpfe prekarisierter EU-Bürger*innen in Deutschland. Als Arbeitsmigrant*innen ringen sie vor dem Hintergrund sozialstaatlicher Ausschlüsse und rassistisch-klassistischer Diskriminierungen auf Märkten um basale Rechte: zum Beispiel um den Zugang zu Wohnraum oder Arbeit. Dabei kämpfen sie nicht zuletzt mit Behörden. Denn die gesellschaftliche Unerwünschtheit der prekarisierten Migrant*innen - im Mediendiskurs ,Armutszuwanderer - mündet beispielsweise in restriktiver Auslegung von Sozialleistungsansprüchen. Auch die Polizei agiert repressiver und verteilt verstärkt Platzverweise auf dem Freilufttagelöhner*innenmarkt (,Arbeitsstrich` für alle, die Sexarbeit nicht abwerten).

Diese behördlichen Praktiken und Formen der Gegenwehr untersucht Riedner auf der Basis einer langjährigen teilnehmenden Beobachtung bei dem Workers' Center. In diesem war sie als Forscherin und Aktivistin zugleich tätig. Sie brachte ihre Arbeit und Expertise ein, während sie die dortige Tätigkeit wissenschaftlich reflektierte. Damit geht die Arbeit weit über eine reine Nabelschau und Analyse sozialer Bewegungspraxen hinaus und untersucht aus der Perspektive des aktivistischen Forschens auch Behördenhandeln. Denn das Center bot Migrant*innen Unterstützung beim Einfordern ihrer Rechte und begleitete sie zum Beispiel bei Behördengängen. 
Gestützt auf diese aktivistische Ethnographie zeichnet die Autorin nach, wie das lokale Migrationsregime in alltäglichen Aushandlungen (re-)produziert wird und dass es dabei stets umkämpft bleibt. Sie zeigt, dass es insbesondere die Ebene der Stadt beziehungsweise der Kommune ist, auf der eine neue Migrationspolitik, die auf territorialem Einschluss bei wohlfahrtsstaatlichem Ausschluss beruht, sowohl entstanden ist als auch in Frage gestellt wird.

Die Arbeit kontextualisiert die neuen Ausschlüsse von Sozialleistungen, welche die Kommunen in Reaktion auf die EU-Freizügigkeit vor allem von Rumän*innen und Bulgar*innen um- und durchsetzten, durch eine Auseinandersetzung mit Regimen der Migration. Diese fasst Riedner nicht nur sehr weit - indem sie sowohl rassistische Repräsentationsregime berücksichtigt als auch Gesetze und Verwaltungspraxen analysiert -, sondern sie analysiert sie auch aus radikal-konstruktivistischer Perspektive. Das heißt, sie versteht Kategorien wie Migration als Aushandlungsergebnisse statt als objektive Tatsachen.

Riedner betont die neoliberale Umgestaltung der Migrationsregime. Neoliberale Stadtpolitiken unterscheiden zunehmend, gute und ,schlechte' Migration: So inkludieren etwa Vielfaltspolitiken eine inwertsetzbare Migration, während die Sicherheitspolitiken der neoliberalen Stadt Arme, gerade auch migrantische Arme, verstärkt unsichtbar machen sollen. Im stadtentwicklungspolitischen Diskurs Deutschlands gelten ethnische Ökonomien oder hochqualifizierte Arbeitskräfte seit der Jahrtausendwende zunehmend als erwünscht. Demgegenüber diskutierten insbesondere die CDU/CSU und Kommunalvertretungen von Städten mit starker Einwanderung aus Osteuropa prekarisierte Arbeitsmigrant*innen als Problem. Dies geschah ab 2013 unter dem Stichwort der ,Armutszuwanderung'. Riedner zeigt, dass die Prekarisierten, die der konservative Diskurs als ,Sozialstaatstouristen“ diffamiert, meist ,arbeitende Arme' sind. Sie problematisiert aber zugleich den lohnarbeitsfreundlichen Bewegungsdiskurs - auch ihres eigenen Workers' Centers -, der auf diese Abwertungen reagiert und den Wunsch der Migrant*innen nach Erwerbsarbeit hervorhebt. Vor allem aber verdeutlicht sie, wie die Armut - insbesondere auf Betreiben der Kommunen - in Deutschland hergestellt wird.

Ein Überblick über die rechtliche Situation der EU-Bürger*innen arbeitet klar heraus, dass oft kommunale Interventionen den Anlass für bundesweite Gesetzesverschärfungen und restriktivere EU-Rechtsauslegungen gaben: vor allem ein Papier des Deutschen Städtetags und Brandbriefe von Großstadtkommunen. Zudem erprobten Kommunen, wie Riedner anknüpfend an eine Überblicksstudie (Spencer/Price 2014) erstmals detailliert am Beispiel Münchens zeigen kann, exkludierende Verwaltungspraxen: zum Beispiel, wenn Sozialämter und Jobcenter Migrant*innen hohe Hürden für den Nachweis ihrer Mittellosigkeit auferlegen.

Im Effekt ist der Sozialleistungszugang von EU-Bürger*innen in Deutschland immer stärker an Arbeit gekoppelt. Riedner interpretiert diesen neuen Trend als ,Aktivierung', also als verstärkte Kommodifizierung der Arbeitskraft. Denn ein System, das Menschen das Existenzminimum versagt, drängt diese nicht nur in Obdachlosigkeit oder prekäres Wohnen. Vielmehr nötigt es sie auch dazu, jedwede Arbeit anzunehmen und gegebenenfalls auch Unterschreitungen von Arbeitsstandards in Kauf zu nehmen. 
In zwei Fallstudien zu Wohnen und Arbeit beschreibt Riedner - in ethnographischem Detailreichtum - dieses System. Sie skizziert die zum Teil rechtswidrigen Versuche der Stadt München, die unerwünschten ,Armutszuwanderer' dergestalt repressiv zu verwalten, dass sie möglichst wenig Kosten verursachen und (vermeintlich) von der Zuwanderung in die Stadt abgeschreckt werden. Im Feld des Wohnens bürdete etwa das Amt für Wohnen und Migration den obdachlosen Migrant*innen den kaum zu erbringenden Nachweis auf, auch im Heimatland nicht über Wohnraum zu verfügen. Auch fühlte sich die Stadt nur für Obdachlose, die mindestens sechs Monate in München gemeldet waren, zuständig. Die Regelungen erwiesen sich zwar bald als rechtlich nicht haltbar und wurden durch einen kollektiven Amtsbesuch der Aktivist*innen des Workers' Center in Frage gestellt. Dennoch verwehr(t)en solche behördlichen Praktiken zunächst den Zugang zu Wohnraum.

Bemerkenswert an der Arbeit ist, dass sie - trotz der Befassung mit extrem prekarisierten Menschen - sehr konsequent die untersuchten Subjekte einbezog und diese mit der Forschung unterstützte. Dies ist keineswegs eine Selbstverständlichkeit - fokussieren doch viele Studien aus dem Feld der Marginalitätsforschung die Institutionen der Marginalisierung oder sie kämpfen, soweit sie Bewegungen Marginalisierter berücksichtigen wollen, mit mangelnden Feldzugängen, Stellvertreterproblemen oder schlicht fehlenden Bewegungen. Riedner hingegen schuf die Bewegung durch das Workers Center, das basisdemokratisch organisiert ist und an den Alltagserfahrungen der Arbeiter*innen ansetzt, kurzerhand selbst. In der Stadtforschung können solche teilnehmenden Beobachtungen, die nicht nur Bewegungsforschung betreiben, sondern aus der Perspektive des Kampfes gegen Amtshandlungen und -willkür auch Verwaltungsethnographien leisten, ein Vorbild sein.

Entsprechend dem aktivistischen Impetus prangert Riedner klar den rassistischen Gehalt des Münchner Migrationsregimes an und kommt zum Schluss:

„Ganz offene Formen des antimigrantischen und antiziganistischen Rassismus verschränkten sich dabei in einer Assemblage des Rassismus [...] mit postliberalen Spielarten des Rassismus, die scheinbar liberale und vernünftige Argumente und eine universelle, selektierende und individualisierende Rationalität einbrachten, die Vielfalt affirmierte, aber zwischen guten und schlechten Migrant*innen unterschied.“ (310)

Obgleich Riedner die Aktivierung zur Arbeit herausarbeitet, stehen weitere Herrschaftsverhältnisse etwas weniger deutlich im Fokus der durchaus intersektional angelegten Arbeit. Bisweilen wird als Rassismus vereindeutigt, was - angesichts der Verknüpfungen von Klasse und Ethnie im ,Armutsmigrationsdiskurs' - ebenso als Klassismus lesbar wäre. So interpretiert Riedner etwa Skandalisierungen eines ungepflegten Aussehens oder Referenzen auf „Müll, unzivilisiertes Verhalten, unkontrollierte[n] Konsum von Zigaretten und Alkohol und die Verdrängung der ,normalen Passant*innen“ (165), die in städtischen Ordnungsdiskursen häufig invoziert werden und ganz unterschiedliche Herrschaftsverhältnisse stützen können, zum Teil auch dort als Rassismus, wo in Zitaten selbst keine ethnischen Kategorisierungen vorgenommen werden. Das verweist darauf, dass eine intensivierte Zusammenarbeit radikal-konstruktivistischer 
Migrationsforschung mit einer stärker marxistisch inspirierten Stadtforschung fruchtbar sein könnte.

Davon unbenommen leistet die Arbeit einen wichtigen Beitrag zur Stadt- und Migrationsforschung und ist daher für Wissenschaftler*innen und Aktivist*innen aus beiden Themenfeldern gleichermaßen relevant. In der Stadtforschung ergänzt sie Arbeiten, die neue Praktiken der Inklusion von Migrant*innen in der neoliberalen ,Stadt der Vielfalt (Rodatz 2018) konstatieren, um einen Blick auf sich parallel entwickelnde neue Ausschlüsse und Prekaritätsformen. Zugleich erweitert sie mit der Thematisierung prekärer Arbeitsmigration in einer Zeit, in der kriegs- und klimabedingte Fluchtmigration stark im Fokus steht, den Blick um eine ,vergessene' Gruppe. In der Migrationsforschung kann die Arbeit an eine lange Tradition anknüpfen, ethnische und nationale Differenzen als konstitutive Elemente eines racial capitalism (Robinson 1983) zu analysieren und jüngst auch ,Migrationsmanagement ${ }^{\star}$ als Teil einer intersektionalen Kapitalismusanalyse zu untersuchen (z. B. Buckel 2012). Riedner ergänzt die bisherigen Forschungen zu expliziten Strategien der Inwertsetzung erwünschter Migration (zum Beispiel durch Anwerbung von Hochqualifizierten oder Pflegekräften). Dies tut sie nicht nur durch eine Analyse der - bisweilen gar nicht intendierten - Inwertsetzung ,unerwünschter' Migration. Vielmehr zeigt sie dabei auch neue, zum Teil paradoxe Mechanismen auf: „Die Ausländerbehörde wurde zur Aktivierungsinstanz und das Jobcenter zur (internen) Grenzbehörde.“ (306)

Dieser Artikel wurde durch das Programm Open Access Publizieren der Deutschen Forschungsgemeinschaft und den Publikationsfonds der Humboldt-Universität zu Berlin gefördert.

\section{Autor_innen}

Jenny Künkel betreibt kritische Sozialforschung zwischen Politikwissenschaften, Geographie, Kriminologie und Queer Studies.

jenny.lena.kuenkel@geo.hu-berlin.de

\section{Literatur}

Buckel, Sonja (2012): Managing Migration. Eine intersektionale Kapitalismusanalyse am Beispiel der Europäischen Migrationspolitik. In: Berliner Journal für Soziologie 22/1. 79-100.

Riedner, Lisa (2018): Arbeit! Wohnen! Urbane Auseinandersetzungen um EU-Migration. Eine Untersuchung zwischen Wissenschaft und Aktivismus. Münster: edition assemblage.

Robinson, Cedric (1983): Black Marxism. The Making of the Black Radical Tradition. London: Zed Press.

Rodatz, Mathias (2017): Neue urbane Ordnungen der Migration. Staatsrassismus in der neoliberalen ,Stadt der Vielfalt‘. Bielefeld: transcript.

Spencer, Sarah / Price, Jonathan (2014): City-Level Responses to Migrant Families with Restricted Access to Welfare Benefits. A European Pilot Study. https://www.compas.ox.ac. uk/media/PR-2014-No_Recourse_Public_Funds.pdf (letzter Zugriff am 8.10.2019). 